\title{
Gynecologic and Breast Oncology Genetic Counseling Program at a Private Hospital in Argentina: Our Experience
}

HOSPITAL ITALIANO

de Buenos Aires
Riggi $M C^{(1,2,3)}$, Noll F (1), Zamora $L^{(1,3)}$, Vaccaro $C^{(2)}$, Perrotta $M^{(1)}$

1) Gynecologic Oncology Service, Department of Gynecology, Hospital Italiano de Buenos Aires

(2) Hereditary Cancer Program (Pro.Can.He), Hospital Italiano de Buenos Aires

(3) Breast Unit, Department of Gynecology, Hospital Italiano de Buenos Aires.

Objectives:The Hereditary Cancer Program of the Hospital Italiano at Buenos Aires is a multidisciplinary team dedicated to the guidance, counseling and detection of hereditary cancer. The team offers patient care, research and provides outreach and education for health workers and patients. Our aim was to describe the characteristics of the patients who were referred to the Breast and Gynecologic Hereditary Cancer Program between January 2014 and July 2016.

Methods: This is a retrospective, descriptive observational study. We obtained information from the electronic clinical records of all patients who were counseled within the program between January 2014 and July 2016 . Here we describe their demographic characteristics as well as types of cancer and availability of genetic testing.

Results: A total of 292 patients were referred for evaluation of breast and gynecologic cancer risk. The reason for referral was: a personal history of cancer (with or without family history) in 135 patients (46,2\%), family history only in 133 patients $(45,5 \%)$, presence of a known mutation within the family in 15 patients $(5.1 \%)$, and personal interest in 9 patients (3\%).

Among all patients, 36 (12.3\%) were of Ashkenazi descent, and $86.6 \%$ met at least one criteria for genetic testing according to NCCN guidelines. Genetic testing was offered to 157 patients (53.8\%). A total of 159 genetics test were performed ( 2 patients had both ashkenazi panel and complete sequencing), 124 were full sequencing of BRCA 1 and 2, 26 were Ashkenazi panel tests and 9 searched for specific known family mutations. Mutations in BRCA 1 or 2 were identiied in $24.6 \%$ of the concluded tests and variants of undetermined significance (VUS) were encountered in $4.5 \%$ of tests.

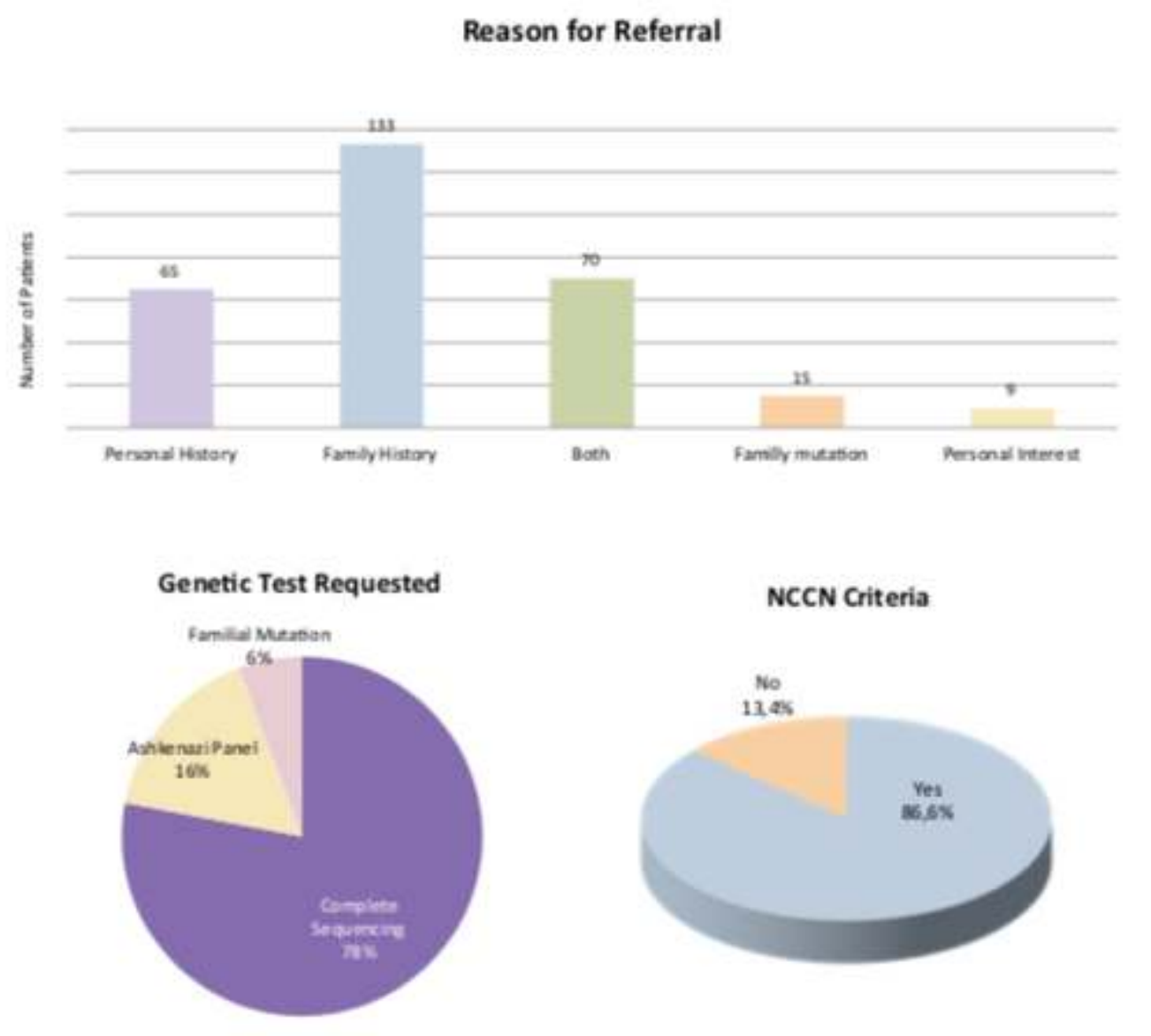

Personal history of patients with concluded genetic test

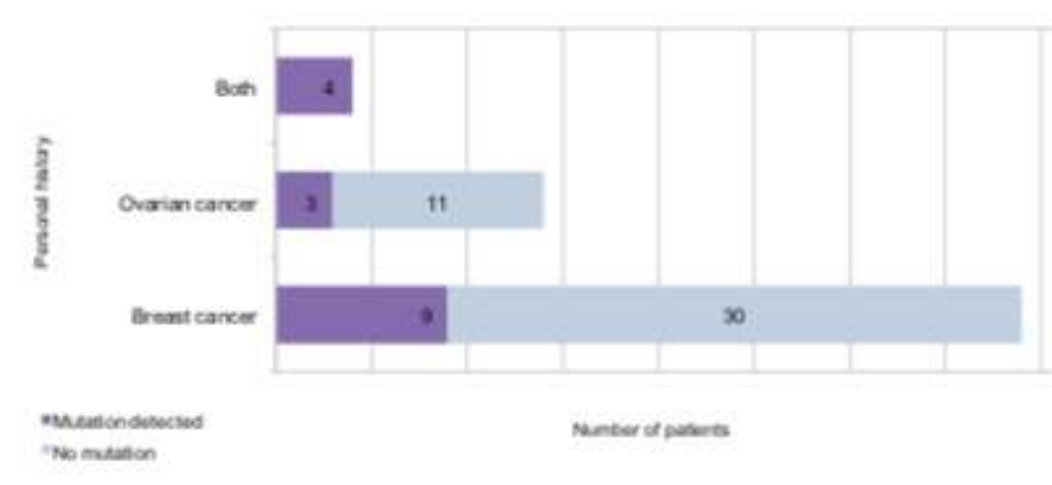

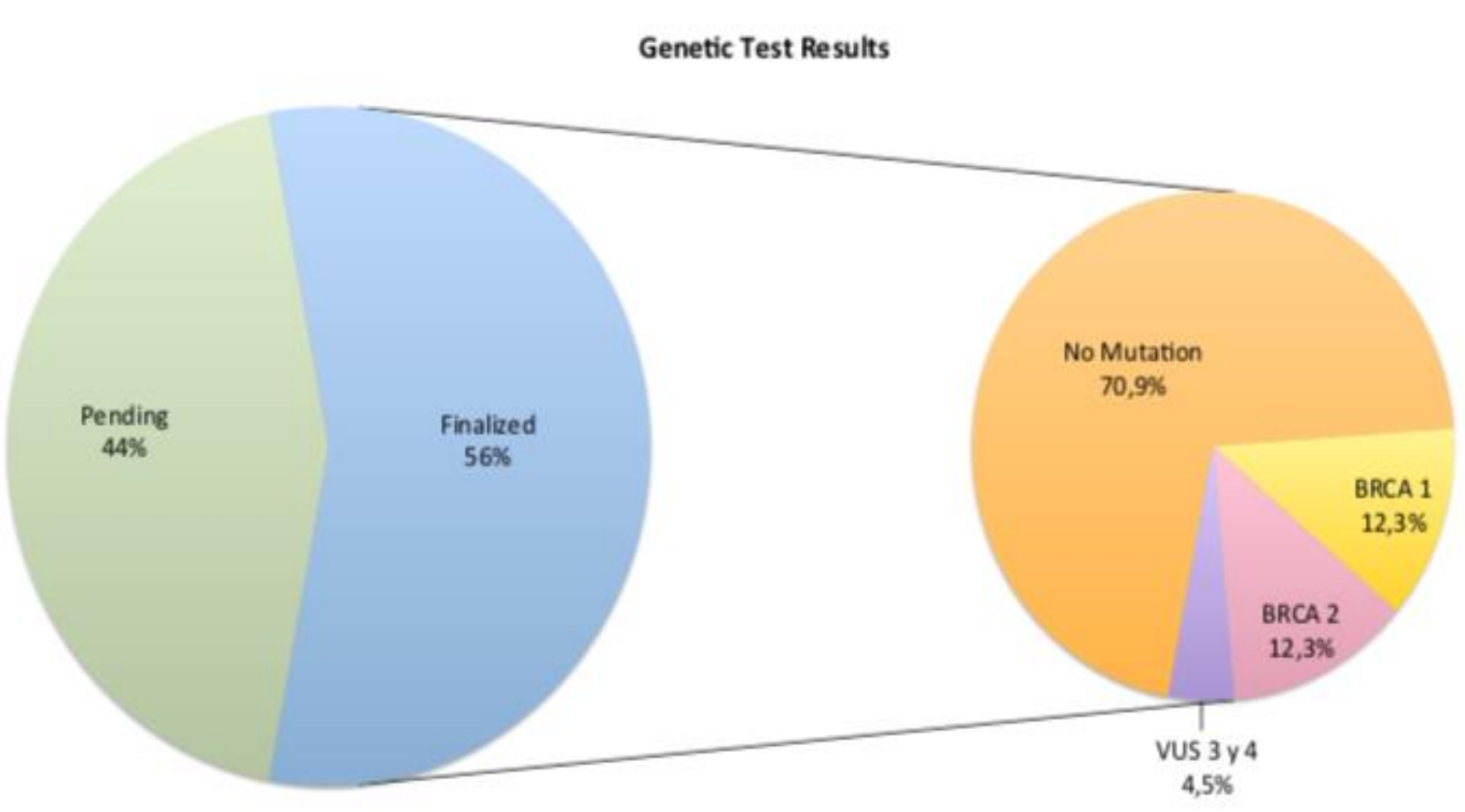

Pathogenic Variants

\begin{tabular}{|c|c|c|c|}
\hline Gender & Genetic Test & BRCA & Mutation \\
\hline Female & Ashkenazi panel & 1 & c.68_69delAG (p.Glu23Valfs) \\
\hline Female & $\begin{array}{l}\text { Complete } \\
\text { sequencing }\end{array}$ & 1 & c.4964_4982del19 (p.Ser1655Tyrfs*16) \\
\hline Female & $\begin{array}{c}\text { Complete } \\
\text { sequencing }\end{array}$ & 1 & c.4964_4982del119 (p.Ser1655Tyrfs*16) \\
\hline Female & $\begin{array}{l}\text { Complete } \\
\text { sequencing }\end{array}$ & 1 & $\begin{array}{c}\text { c.4128_2129delAA (p.Thr1376- } \\
\text { Ser1377ThrArgfs) }\end{array}$ \\
\hline Female & $\begin{array}{l}\text { Complete } \\
\text { sequencing }\end{array}$ & 1 & c.5434C>G (p.Pro1812Ala) \\
\hline Female & $\begin{array}{l}\text { Complete } \\
\text { sequencing }\end{array}$ & 1 & c.3706_3707delAA (p.Asn1236Tyrfs) \\
\hline Female & $\begin{array}{l}\text { Complete } \\
\text { sequencing }\end{array}$ & 1 & c.4687delT(p.Tyr1563fs fs*38) \\
\hline Female & $\begin{array}{l}\text { Complete } \\
\text { sequencing }\end{array}$ & 1 & c.1360_1361del AG (p.Ser454Terfs)* \\
\hline Female & Familial mutation & 1 & c.1360_1361delAG (p.Ser454Terfs) \\
\hline Female & Familial mutation & 1 & c.5123 C>A (p.Ala1708Glu) \\
\hline Male & Familial mutation & 1 & c.4042G >T (p.Gly1348Ter) \\
\hline Female & Ashkenazi panel & 2 & c.2808_2811delACAA (p.Ala938Profs) \\
\hline Female & Ashkenazi panel & 2 & c.5946delT (p.Ser1982Argfs) \\
\hline Female & Ashkenazi panel & 2 & c.5946delT (p.Ser1982Argfs) \\
\hline Male & Ashkenazi panel & 2 & c.5946delT(p.Ser1982Argfs) \\
\hline Female & $\begin{array}{l}\text { Complete } \\
\text { sequencing }\end{array}$ & 2 & c.5946delT (p.Ser1982Argfs) \\
\hline Female & $\begin{array}{l}\text { Complete } \\
\text { sequencing }\end{array}$ & 2 & c.5351dupA (p.Asn1784Lysfs) \\
\hline Female & $\begin{array}{l}\text { Complete } \\
\text { sequencing }\end{array}$ & 2 & c.2656delA (p.N886fs*9)* \\
\hline Female & $\begin{array}{l}\text { Complete } \\
\text { sequencing }\end{array}$ & 2 & c.1670T>G (p.Leu557Ter) \\
\hline Female & Familial mutation & 2 & c.5669_5673delTGGCA (p.Met1890Argfs) \\
\hline Female & Familial mutation & 2 & c.1670T>G (p.Leu557Ter) \\
\hline Female & Familial mutation & 2 & c.2656delA (p.N886fs*9) \\
\hline
\end{tabular}

Personal History (Age at diagnosis) Family Mutation

$$
\begin{aligned}
& \text { Ovarian cancer (55) } \\
& \text { Ovarian cancer (73) } \\
& \text { Breast cancer (49) }
\end{aligned}
$$$$
\text { Breast cancer (49)/Ovarian cancer (59) }
$$$$
\text { Breast cancer (68) }
$$$$
\text { Breast cancer (53) }
$$$$
\text { Breast cancer (38) }
$$$$
\text { Breast cancer (53) }
$$$$
\text { NO }
$$$$
\text { NO }
$$$$
\text { NO }
$$$$
\text { Breast cancer (57)/Ovarian cancer (64) No }
$$$$
\text { NO }
$$$$
\text { Breast cancer (60) /Ovarian cancer (65) }
$$$$
\text { Breast cancer(81) -Melanoma (60) }
$$$$
\text { NO }
$$$$
\text { Breast cancer (50) }
$$$$
\text { Ovarian cancer (52) }
$$$$
\text { Breast cancer (32) }
$$$$
\text { Breast cancer (33) }
$$$$
\text { Breast cancer (65)/Ovarian cancer (66) }
$$$$
\text { NO }
$$

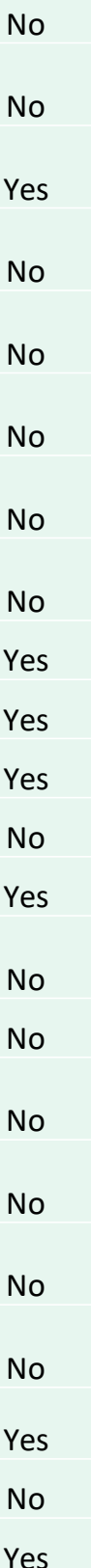

Conclusions: Referral for genetic counseling and molecular testing has increased steadily, probably due to greater knowledge as well as improved accessibility and insurance coverage. It is crucial to continue creating awareness about the importance of diagnosis of cancer predisposition syndromes. 